\title{
A Study of Transconductance Degradation in HEMT Using a Self-consistent Boltzmann-Poisson-Schrödinger Solver
}

\author{
R. KHOIE \\ Department of Electrical and Computer Engineering, University of Nevada, Las Vegas, NV 89154
}

\begin{abstract}
A self-consistent Boltzmann-Poisson-Schrödinger Solver is used to study the transconductance degradation in high electron mobility transistor (HEMT), which has extensively been reported by both experimental [1]-[8] and computational [9]-[13] researchers. As the gate voltage of a HEMT device is increased, its transconductance increases until it reaches a peak value, beyond which, the transconductance is degraded rather sharply with further increase in applied gate bias. We previously reported a two-subband self-consistent Boltzmann-Poisson-Schrödinger Solver for HEMT. [14] We further incorporated an additional self-consistency by calculating field-dependent, energy-dependent intersubband and intrasubband scattering rates due to ionized impurities and polar optical phonons.[15] In this work, we have used our Boltzmann-Poisson-Schrödinger Solver and studied the effects of the intersubband and intrasubband scatterings of electrons, on the transconductance of a single quantum well HEMT device. The results of our simulations exhibit the same pattern reported by others [1]-[13]. We concluded that the degradation of transconductance of the device with applied gate bias is attributed to the increased intersubband and intrasubband scattering of electrons, and hence to the reduction of electrons velocity in the channel.
\end{abstract}

Keywords: Transconductance, Degradation, Poisson, Schrödinger, Boltzmann, Scattering

\section{INTRODUCTION}

Recent advances in III-V compound semiconductor growth techniques have resulted in successful development of high speed devices with transconductances ranging from $427 \mathrm{mS} / \mathrm{mm}$ in a $1 \mu \mathrm{m}$ wide 2-D MESFET [2] to $1740 \mathrm{mS} / \mathrm{mm}$ in a $50 \mathrm{~nm}$ self-aligned-gate pseudomorphic HEMT [8]. These impressive high-frequency parameters have been measured at low gate voltages. In fact, as the gate voltage of a HEMT is increased, the transconductance increases until it reaches a peak value, beyond which, the transconductance is degraded with further increase in applied gate bias.[1]-[13] The reasons cited for this degradation include: increase in gate leakage current [1], reduction of inversion charge in the channel [6], dislocations [7], decrease in the average electron velocity [8], increase in population of electrons in the donor AlGaAs layer [9], and neutralized donor effect [11].

The high transconductance in HEMT is obtained mainly by scaling down the channel length of the device to $500 A^{\circ}$. Further reduction in the gate length results in degradation of the transconductance and decrease in the cut-off frequency. Kizilyalli, et. al.[9] have performed a Monte Carlo study of short channel 
effects in a submicrometer AlGaAs/GaAs HEMT, and suggested that the high-frequency performance of the device can be improved by scaling the gate length down to a minimum of about $0.1 \mu \mathrm{m}$, beyond which the device transconductance is degraded rapidly. They discussed reasons for observed degradation of the transconductance of ultra-small device, citing poor charge control in the channel, and sharp reduction in the output resistance of the device as the main sources of transconductance degradation.

We previously reported a two-subband self-consistent Boltzmann-Poisson-Schrödinger Solver for high electron mobility transistor, in which we self-consistently solved the two higher moments of Boltzmann equation, along with Poisson and Schrödinger equations.[14] We further incorporated an additional self-consistency by calculating field-dependent, energy-dependent intersubband and intrasubband scattering rates due to ionized impurities and polar optical phonons.[15] In this paper, we have used our Boltzmann-Poisson-Schrödinger Solver and studied the effects of the intersubband and intrasubband scatterings of electrons, on the transconductance of a single quantum well HEMT device. We have performed simulations on a $0.5 \mu m$ HEMT, with and without the energy-dependent field-dependent scattering rates. The comparison between the resulting $g_{m}-V_{G}$ curves is used to attribute the transconductance degradation to the scattering of electrons by polar optical phonons and ionized impurities.

\section{BOLTZMANN-POISSON-SCHRÖDINGER SOLVER AND SCATTERING RATES}

The Boltzmann-Poisson-Schrödinger Solver consists of the two higher moments of Boltzmann equation, Poisson and Schrödinger equations self-consistently solved for electrons in the first subband, second subband, and the bulk system (electrons residing in the third and higher subbands, with no quantum restriction in their motion). An additional self-consistency is added by calculating the intersubband and intrasubband scattering rates of electrons by polar optical phonons and ionized impurities. The rates of transfer of electrons and their energies to and from each subband are calculated from the intersubband and intrasubband scattering rates, and are used in a self-consistent manner in the Boltzmann-Poisson-Schrödinger Solver. The two higher moments of Boltzmann equation written for the first two subbands are:[14]

$$
\begin{aligned}
& \frac{\partial\left(n_{i}(x, t)\right)}{\partial t}=\nabla .\left(-\mu_{i} n_{i}(x, t) \nabla V(x)+\nabla\left(D_{i} n_{i}(x, t)\right)\right) \\
& +\sum_{j \neq i}\left(\frac{n_{j-}-n_{o j}}{\tau_{j i}}\right)-\sum_{j \neq i}\left(\frac{n_{i-} n_{o i}}{\tau_{i j}}\right) \quad i, j=1,2 \\
& \frac{\partial\left(n_{i}(x, t) E_{i}(x, t)\right)}{\partial t}=-J . \nabla V(x)+ \\
& \nabla .\left(-\mu_{E, i} n_{i}(x, t) E_{i}(x, t) \nabla V(x)+\nabla\left(D_{E, i} n_{i}(x, t) E_{i}(x, t)\right)\right) \\
& +\sum_{j \neq i}\left(\frac{n_{j} E_{j}-n_{j o} E_{o j}}{\tau_{E, j i}}\right)-\sum_{j \neq i}\left(\frac{n_{i} E_{i}-n_{i o} E_{o i}}{\tau_{E, i j}}\right) \\
& -\sum_{j \neq i}\left(\frac{n_{j}-n_{o j}}{\tau_{j i}}\right) \hbar \omega_{0} \quad i, j=1,2
\end{aligned}
$$

Similar equations are written for the electrons in the third and higher subbands, which are considered as bulk three dimensional electrons with no quantum restriction in their motion. In the above equations $i, j=1,2$ refers to the first and second subbands, respectively. $n$ is the electron concentration, $J$ is the electron current density, $q$ is the electronic charge, $\mu_{\mathrm{i}}$ is the mobility, $D_{i}$ is diffusivity, $\mu_{E, i}$ is flux mobility, $D_{E, i}$ is the flux diffusivity, $E_{i}$ is the average electron energy, $\tau_{i j}$ is the electron relaxation time, $\tau_{E, i j}$ is energy relaxation time for electrons scattered from subband $i$ to subband $j$, and $\omega_{0}$ is the polar optical phonon frequency. The summation terms $\left(\Sigma_{j} \neq_{i}\left(\frac{n_{j}-n_{o j}}{\tau_{j i}}\right)\right)$ and $\left(-\Sigma_{j} \neq_{i}\left(\frac{n_{i}-n_{o i}}{\tau_{i j}}\right)\right)$ in Eq. (1), account for the scattering of electrons from subband $j$ to subband $i$, and back, respectively. The summation term $\left(\Sigma_{j} \neq_{i}\left(\frac{n_{j} E_{j}-n_{j o} E_{o j}}{\tau_{E, j i}}\right)\right)$ and $\left(-\Sigma_{j} \neq_{i}\left(\frac{n_{i} E_{i}-n_{i o} E_{i o}}{\tau_{E, i j}}\right)\right)$ in Eq. (2) account for the energy gained (or lost) due 
to the scattering of electrons from subband $j$ to subband $i$, and back, respectively. The last summation term $\left(-\Sigma_{j} \neq_{i}\left(\frac{n_{j}-n_{o j}}{\tau_{j i}} \hbar \omega_{0}\right)\right.$, in Eq. (2) includes the effect of the loss of energy by the electron to the polar optical phonons. The relaxation times $\tau_{i j}$ and $\tau_{E, i j}$ for electrons and their energies are calculated from the rates of scattering of electrons by polar optical phonons and ionized impurities. Details of these calculations are given in [15].

The remaining components of the Boltzmann-Poisson-Schrödinger Solver are:

$$
\frac{\partial^{2} V}{\partial x^{2}}+\frac{\partial^{2} V}{\partial y^{2}}=-\frac{q}{\varepsilon}\left[N_{D}(x, y)-n(x, y)\right]
$$

and

$$
-\frac{\hbar^{2}}{2 m^{*}} \frac{d^{2} \psi_{i}(x)}{d x^{2}}-q V(x, y) \psi_{i}(x)=E_{i} \psi_{i}(x)
$$

where $q$ is the electronic charge, $\varepsilon$ is the dielectric constant, $n$ is the total electron concentration, $N_{D}$ is the doping level, $m^{*}$ is the electron effective mass, $V(x, y)$ is the electrostatic potential, and $\mathrm{V}_{i}$ is the wavefunction corresponding to the eigenenergy $E_{i}$ for the $i$-th subband.

\section{RESULTS}

We have simulated a HEMT device with $0.5 \mu \mathrm{m}$ gate length, $60 \mathrm{~nm}$ wide $A l G a A s$ layer doped with $5.0 \times 10^{17} / c c$ impurities, all on a GaAs layer doped with $10^{14} / c c$ impurities. Using our Boltzmann-Poisson-Schrödinger Solver, two sets of simulations are performed. First for a one-subband quantum well in which only the electrons in the lowest subband are forming the two dimensional electron gas, and the electrons in the second and higher subbands are three dimensional carriers without being subject to intersubband and intrasubband scattering mechanisms. Second set of data are produced for the same device, but with the electrons in the first and second subbands forming the two dimensional gas with quantum restriction in their motion and subject to the intersubband and intrasubband scattering due to both polar

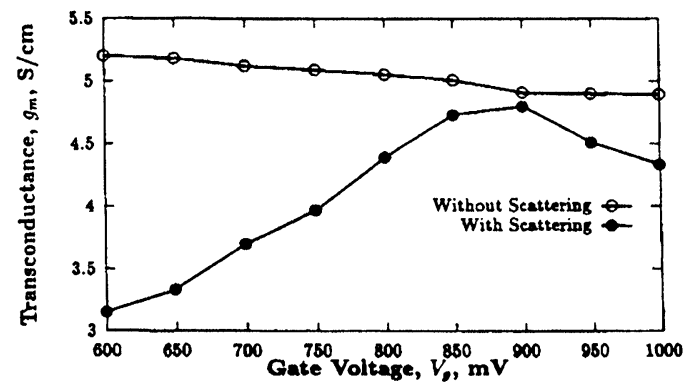

FIGURE 1 Transconductance as a function of gate bias in a HEMT device calculated by our Boltzmann-Poisson-Schrödinger Solver $(\bullet)$ with self-consistent scattering rates $(0)$ without scattering rates

optical phonons and ionized impurities. In both sets of simulations, drain voltage, $V_{D}$ was kept at $1.0 \mathrm{~V}$, which is somewhat below the pinch-off voltage of the device. Although the Boltzmann-Poisson-Schrödinger Solver produces the electrostatic potentials, eigenfunctions, eigenenergies, electron concentration, and electron energy in all three subbands, (see [14]-[15]), as well as all terminal characteristics of the device, in this paper we only show the variations of transconductance with gate voltage, which are shown in Fig. (1)for both sets of data. The value of transconductance, with the one-subband model, and without the scattering rates included, decreases slightly (and almost linearly) as the gate voltage is varied over the range of $0.6 \mathrm{~V}$ to $1.0 \mathrm{~V}$, whereas with the two-subband model and the scattering rates included, the transconductance increases at first, reaching a peak value of about $470 \mathrm{mS} / \mathrm{mm}$ at $0.9 \mathrm{~V}$, and then decreasing beyond that point. Obviously, the pattern of the second set of data is closer to the experimental results reported by [1] and [8], which are shown in Fig. (2).

\section{CONCLUSIONS}

The transconductance degradation of HEMT device at high gate voltages is studied using a Boltzmann-Poisson-Schrödinger Solver. The results of our simulations compares well to those experimentally measured [1]-[8], and theoretically computed [9]-[13]. Although, precise comparison between our 


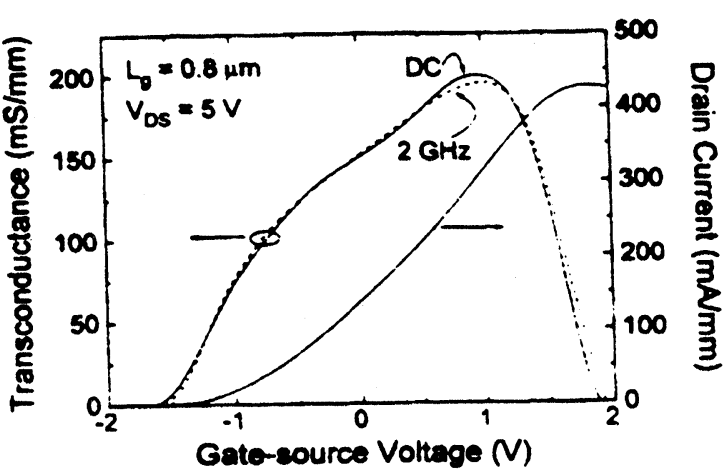

(a)

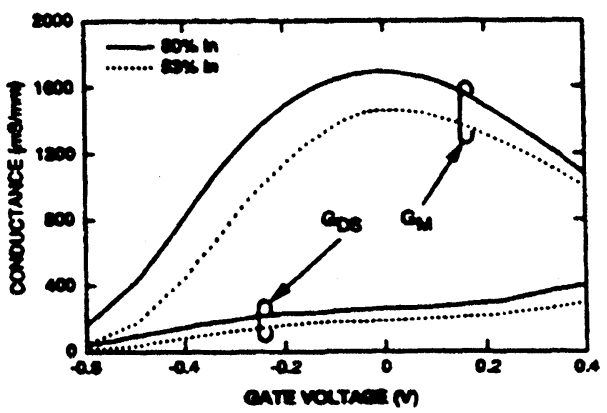

(b)

FIGURE 2 (a) Transconductance as a function of gate voltage of (a) a $0.8 \mu \mathrm{m} I n_{.53} \mathrm{Ga}{ }_{.47} \mathrm{As}$-InP HFET device reported by [1], and (b) a $50 \mathrm{~nm}$ self-aligned pseudomorphic AlInAs-GaInAs HEMT reported by [8]

simulation results and those reported by [1]-[13] could not be made due to the difference in device parameters, the pattern of transconductance degradation at high gate voltages is obvious in these data; the transconductance increases until it reaches a peak value, beyond which, the transconductance is degraded with further increase in applied gate bias. The variety of reasons cited for this degradation is as great as the number of the researchers reporting them. We found the following arguments to be the most compelling: increase in gate leakage current [1], reduction of inversion charge in the channel [6], dislocations [7], decrease in the average electron velocity [8], increase in population of electrons in the donor AlGaAs layer [9], and neutralized donor effect [11]. The results of our simulation support the reason cited by [8], namely the decrease in the electron velocity due to increase in the rate of scattering of carriers by polar optical phonons, and ionized impurities.

\section{References}

[1] D. Greenberg, J. Del Alamo, R. Bhat, "Impact lonization and Transport in InAlAs $/ n^{+}$- InP HFET", IEEE Trans. Electron Devices, Vol. 42, No. 9, pp. 1574-1582, 1995.

[2] W. Peatman, M. Hurt, H. Park, T. Ytterdal, R. Tsai, M. Shur, "Narrow Channel 2-D MESFET for Low Power Electronics", IEEE Trans. Electron Devices, Vol. 42, No. 9, pp. 1569-1573, 1995.

[3] H. Fawaz, J. Gest, J. Zimmermann, "A Novel Model of the Gate Current in Heterojunction FET's," IEEE Trans. Electron Devices, Vol. 40, No. 5, pp. 846-851, 1993.
[4] R. Plana, L. Escotte, O. Llopis, H. Amine, T. Parra, M. Gayral, J. Graffuil, "Noise in Al-GaAs/InGaAs/GaAs Pseudomorphic HEMT's from $10 \mathrm{~Hz}$ to $18 \mathrm{GHz}$ ", IEEE Trans. Electron Devices, Vol. 40, No. 5, pp. 852-858, 1993.

[5] J. Abrokwah, J. Huang, J. Hallmark, "Anisotype-Gate Self-Aligned p-Channel Heterostructure Field Effect Transistors", IEEE Trans. Electron Devices, Vol. 40, No. 2, pp. 278-284, 1993.

[6] S. Sargood, G. Taylor, P. Claisse, T. Vang, P. Cooke, D. Docter, P. Kiely, C. Burrus, "A Quantum Well Inversion Channel Heterostructure as a Multifunctional Component for Optoelectronic Integrated Circuits", IEEE Journal of Quantum Electronics, Vol. 29, No. 1, pp. 136-149, 1993.

[7] G. Wang, Y. Chen, L. Eastman, "A $0.1 \mu \mathrm{m}$ Gate $A l_{0.5} I n_{0,5} A s$ / $G a_{0,5} I n_{0,5}$ As MODFET Fabricated on GaAs Substrate," IEEE Trans. Electron Devices, Vol. 35, No. 7, pp. 818-823, 1988.

[8] L. Nguyen, A. Brown, M. Thompson, L. Jelloian, "50-nm Self-Aligned-Gate Pseudomorphic AllnAs/GalnAs High Electron Mobility Transistors", IEEE Trans. Electron Devices, Vol. 39, No. 9, pp. 2007-2014, 1992.

[9] I. Kizilyalli, M. Artaki, N. Shah, A. Chandra, "Scaling Properties and Short-Channel Effects in Submicrometer AlGaAs/GaAs MODFET's: A Monte Carlo Study", IEEE Trans. Electron Devices, Vol. 40, No. 2, pp. 234-249, 1993.

[10] Y. Zhao, D. Tsui, P. Chao, "Electron Transport in 0.15- $\mu \mathrm{m}$ Gate $\operatorname{In}_{0,52} A l_{0,48} A s / I_{0.53} G a_{0,47} A s$ HEMT", IEEE Trans. Electron Devices, Vol. 40, No. 6, pp. 1067-1070, 1993.

[11] Y. Wang, M. Houng, C. Chu, H. Chen, "Self-consistent Simulation of Modulation Doped Field Effect Transistors", Solid State Electronics, Vol. 37, No. 2, pp. 237-241, 1994.

[12] K. Hida, T. Itoh, K. Ohata, "A Novel 2DEGFET Model Based on the Parabolic Velocity-Field Curve Approximation", IEEE Trans. Electron Devices, Vol. 33, No. 10, pp. 1580-1585, 1986.

[13] K. Liu, A. Anwar, "A Self-consistent Calculation of the Small Signal Parameters for AlGaAs/GaAs and AlGaAs/InGaAs/GaAs HEMTs", Solid State Electronics, Vol. 37, No. 1, pp. 51-54, 1994. 
[14] R. Khoie, H. Arman, "A Two-Subband Self-Consistent Model for High Electron Mobility Transistor Including Intersubband and Intrasubband Scattering Mechanisms", Proceedings of the International Workshop on Computational Electronics, Ed. K. Hess, U. Ravaioli, R. Dutton, pp. 181-184, 1992.

[15] H. Arman, R. Khoie, "A Self-Consistent Multisubband Model for Calculation of Scattering Rates in Quantum Well Structures", Proceedings of the International Workshop on Computational Electronics, Ed. K. Hess, U. Ravaioli, R. Dutton, pp. 175-179, 1992.

\section{Biography}

Rahim Khoie received $\mathrm{Ph} . \mathrm{D}$. degree in Electrical Engineering from University of Pittsburgh, Pennsyl- vania, in 1986. Since then he has been with University of Nevada, Las Vegas, where he has been involved in teaching solid state, semiconductor physics, and electronics courses, and research on compound semiconductors and heterostructure devices. He has published numerous papers in proceedings and journals, and has received several research grants. He is presently working in numerical modeling of compound semiconductor devices such as high electron mobility transistor, resonant tunneling transistor, quantum well laser, and photovoltaics in quantum wells. 

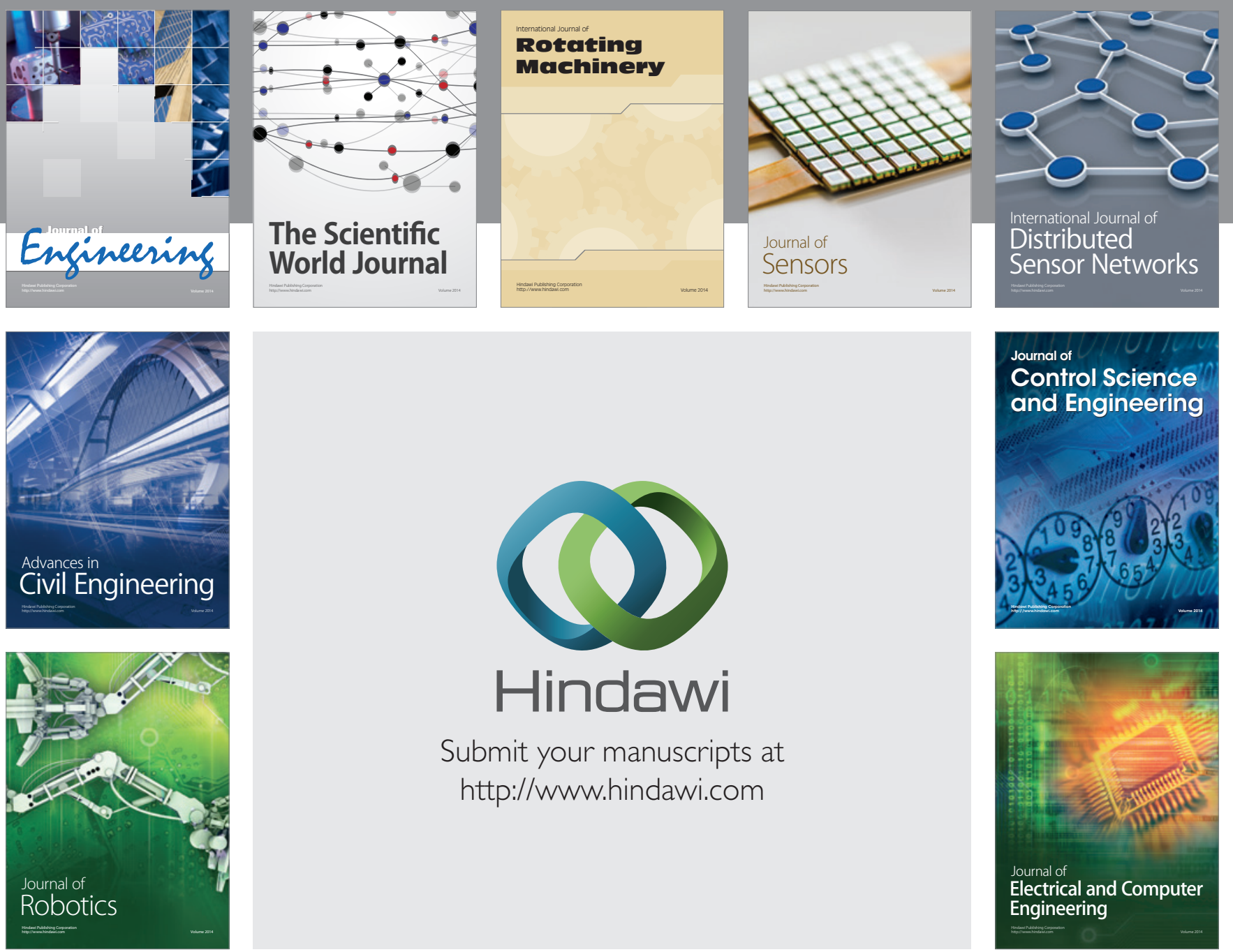

Submit your manuscripts at

http://www.hindawi.com
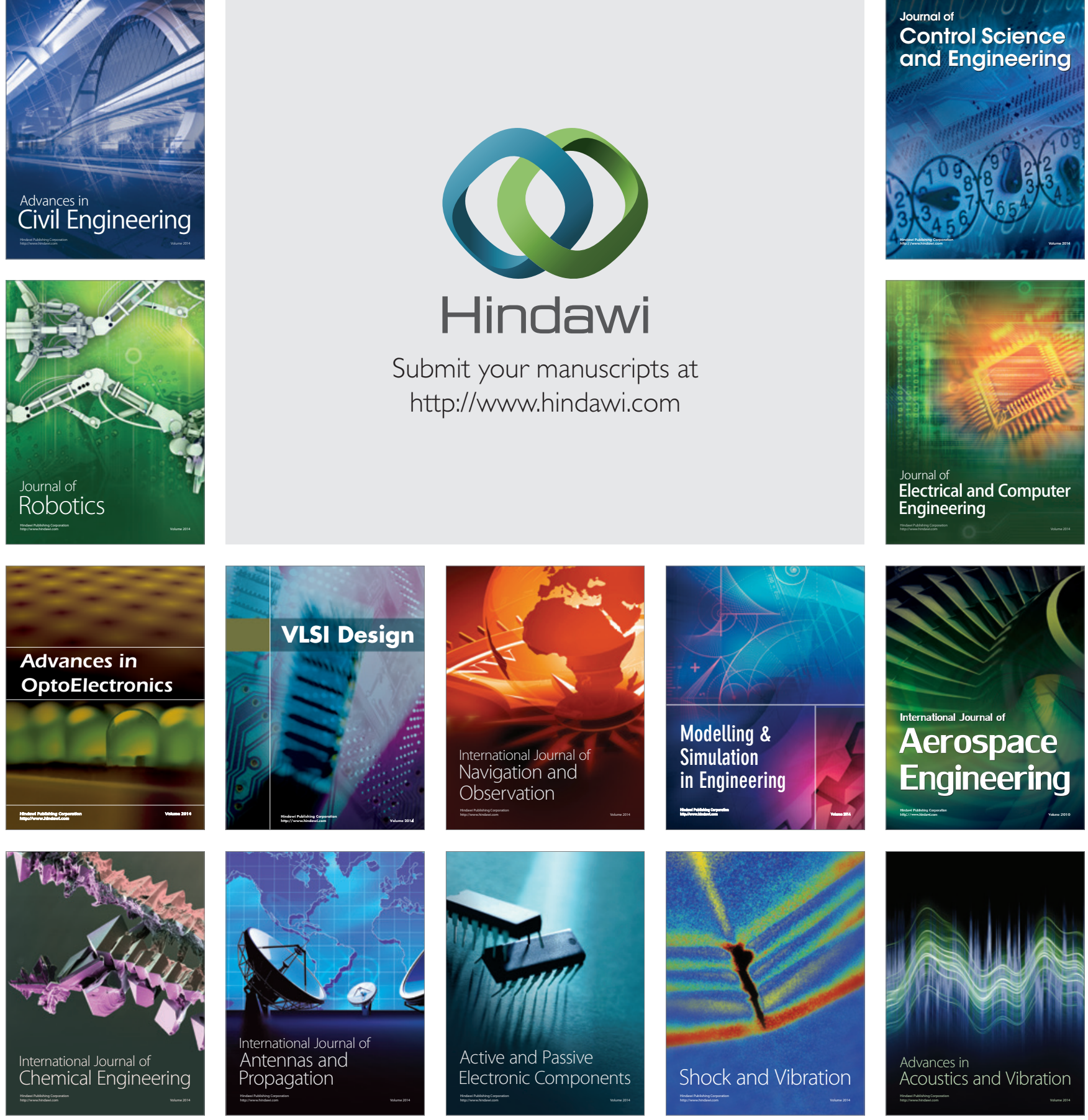